\title{
Study on Constitutive Relation of Viscoelastic Conveyor Belt
}

ISSN: 2578-0255

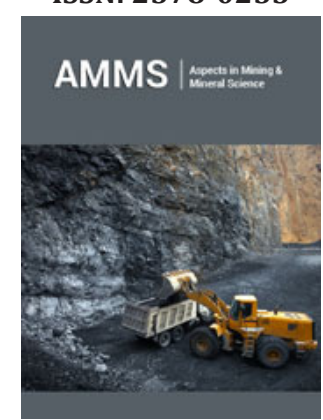

*Corresponding author: Meng Wenjun, School of Mechanical Engineering, Taiyuan University of Science and Technology, Taiyuan, China

Submission: 筒July 15, 2020

Published: 漈 August 27, 2020

Volume 5 - Issue 3

How to cite this article: Wenjun $\mathrm{M}^{*}$, Jinhu S, Biao Z. Study on Constitutive Relation of Viscoelastic Conveyor Belt. Aspects Min Miner Sci. 5(3). AMMS. 000614. 2020.

DOI: 10.31031/AMMS.2020.05.000614

Copyright@ Wenjun M, This article is distributed under the terms of the Creative Commons Attribution 4.0 International License, which permits unrestricted use and redistribution provided that the original author and source are credited.
Wenjun $\mathrm{M}^{*}$, Jinhu S and Biao Z

School of Mechanical Engineering, Taiyuan University of Science and Technology, China

\section{Abstract}

With the development of the economy, conveyors are increasingly moving toward long distances, large volumes, and high belt speeds, and the power of belt conveyors is closely related to the running resistance of conveyors. In the past, the indentation resistance is based on the material properties of the ideal conveyor belt. For example, Maxwell, Kelvin, or the three-parameter model are used for design calculation. The calculation results are still different from the actual ones, especially on the special conditions such as high temperature, high belt speeds. In this paper, the integral constitutive relation which is easier to test and better describe the memory performance of the material of the object after loading is established. It has guiding significance for the design of the belt conveyor.

Keywords: Conveyor belt; Integral constitutive relationship; Viscoelastic

\section{Introduction}

With the development of economy, belt conveyors are increasingly developing toward long distances, large-scale, and high belt speeds. People's requirements for the calculation of belt conveyors are increasing. Among them, the power calculation is closely related to the resistance of the conveyor. The resistance generated during the stable operation of the conveyor includes the indentation rolling resistance (IRR), the running resistance of the conveyor belt around the roller, the impact of the materials and the bending resistance of the conveyor belt [1]. Among them, the indentation rolling resistance is the largest, and the energy it consumes accounts for about $60 \%$ of the total energy consumption of the belt conveyor [2]. Therefore, the accurate prediction and reduction of IRR is the hotspot of current research and the current research [3-5].

Differential constitutive relations are widely used in the early development of viscoelastic theory. It is more convenient to solve certain problems. The quasi-static mechanical behavior of the material can be determined through creep experiments and stress relaxation experiments. It is considered that creep compliance and relaxation modulus are functions of time, which depend on the material itself and have nothing to do with the load frequency, amplitude, etc. [6]. Domestic Lu Yan analyzes the influence of different working conditions elements on the indentation resistance of the conveyor, and establishes a mathematical model [7]; Zhao Xiaoxia and others conduct dynamic and static analysis of the indentation resistance [8]; Hou Hongwei uses the WLF principle Quantitatively describe the indentation resistance and the polymer's kinetic properties, and qualitatively analyze the relationship between indentation rolling resistance and positive pressure, and the thickness of the conveyor belt [9]. Therefore, this article first derives the integral constitutive relationship that is easy to carry out experimental measurements, and then analyzes the indentation state of the conveyor belt to provide a basis for the later conveyor design. 


\section{Integral Constitutive Relationship}

For linear viscoelastic materials, the strain response under step stress $\sigma(t)=\sigma_{0} H(t)$ is expressed as $\sigma(t)=\sigma_{0} J(t)$, as shown in Figure 1(a). Applying the principle of superposition, and so on, if there are $r$ stress increments sequentially acting on the object at a time, Then the total strain at a later time is

$$
\varepsilon(t)=\sigma_{0}+\sum_{i=1}^{r} \Delta \sigma_{i} J\left(t-\xi_{i}\right)
$$

Although the force process of an object is more complicated, it can be seen as a superposition of multiple forces. As shown in Figure 1(b), suppose the stress acting on the object is a continuous differentiable function $\sigma(t)$, and decompose it into the action of $\sigma_{0} H(t)$ and countless very small stresses $d \sigma(\xi) H(t-\xi)$, then the strain response at time $\mathrm{t}$ is:

$$
\varepsilon(t)=\sigma_{0} J(t)+\int_{0^{+}}^{t} J(t-\xi) \frac{d \sigma(\xi)}{d \xi} d \xi
$$

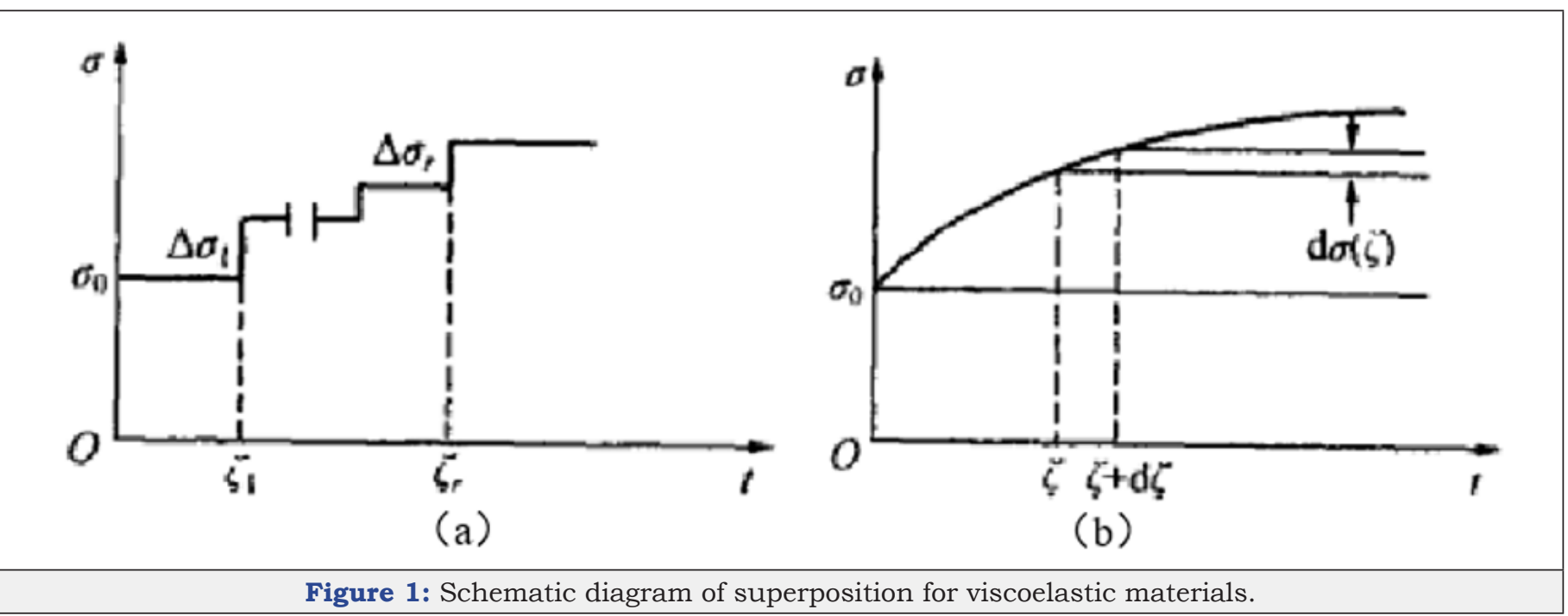

When $t<0, \sigma(t)=0, \quad J(t)<0$.Therefore, formula (2) can be expressed as:

$$
\varepsilon(t)=\int_{-\infty}^{+\infty} J(t-\xi) \sigma(\xi) d \xi
$$

Equation 2 is just the integral constitutive equation. Given the creep function of a material and a given stress $\sigma(t)$, the creep constitutive equation of the material can be obtained. This article selects Burgers four-parameter model. After calculation, there is

$$
J(t)=\frac{1}{E_{1}}+\frac{T}{\eta_{2}}+\frac{1}{E_{3}}\left(1-e^{-t / \tau}\right)
$$

Substituting formula (4) into formula (3), the integral constitutive relationship of conveyor belt based on Burgers fourparameter model is:

$$
\varepsilon(t)=\int_{-\infty}^{+\infty}\left(\frac{1}{E_{1}}+\frac{T}{\eta_{2}}+\frac{1}{E_{3}}\left(1-e^{(\xi-t) / \tau}\right)\right) \sigma(\xi) d \xi
$$

\section{Conclusion}

This paper first derives the integral constitutive relationship of the four-parameter Burgers model of the conveyor belt. Compared with the previous two-parameter or three-parameter constitutive relationship, the new four-parameter integral constitutive relationship can more accurately express the viscoelasticity of the conveyor belt, and it is also easier to conduct experimental measurements. The further study of the four-parameter integral constitutive relationship is of guiding significance for the design and manufacture of belt conveyors since this study will change the selection of various parameters in the design process and affect the use of belt conveyors in mining and other industries.

\section{References}

1. Krol R, Kisielewski W, Kaszuba D, Gładysiewicz L (2017) Testing belt conveyor resistance to motion in underground mine conditions. International Journal of Mining, Reclamation and Environment 31(1): $1-13$.

2. Hager M, Hintz A (1993) Energy-saving design of belts for long conveyor systems. Bulk Solids Handling 13(4): 749-758.

3. Hou YF, Meng QR (2008) Dynamic characteristics of conveyor belts. Journal of China University of Mining and Technology 18(4): 629-633.

4. Li J, Pang X (2018) Belt conveyor dynamic characteristics and influential factors. Shock and Vibration 2018: 1-13.

5. Chen G, Zhang X, Wang ZJ, Li F (2015) An enhanced artificial bee colony-based support vector machine for image-based fault detection. Mathematical Problems in Engineering 2015: 1-12.

6. Yang QT (1992) Viscoelasticity. Wuhan: Huazhong University of Technology Press, China.

7. Hou WH (2019) Analysis and application of compressive resistance characteristics of conveyor belt, coal mine machine. pp. 154-156. 
8. Zhao XX, Meng WJ (2018) Research on static indentation depth and dynamic indentation rolling resistance of conveyor belt, coal mine safety. 1: 133-135.
9. Yan Lu (2017) Research on the influence of working conditions on the indentation resistance of belt conveyor.

For possible submissions Click below:

\section{Submit Article}

